\title{
ON A TWO LAG DIFFERENTIAL DELAY EQUATION
}

\author{
R. D. BRADDOCK ${ }^{1}$ and P. VAN DEN DRIESSCHE ${ }^{2}$
}

(Received 16 January 1981; revised 10 March 1982)

\begin{abstract}
The non-linear differential difference equation of the form

$$
\frac{d N}{d t}=R N(t)\left(1-B N\left(t-T_{1}\right)-C N\left(t-T_{2}\right)\right)
$$

is investigated. This equation, with constant coefficients, is used to model the population level, $N$, of a single species, and incorporates two constant time lags $T_{2}>T_{1}>0$; for example, regeneration and reproductive lags. The linear equation is investigated analytically, and some linear stability regions are described. The special case in which the two delay terms are equally important in self damping, $B=C$, is investigated in detail. Numerical solutions for this case show stable limit cycles, with multiple loops appearing when $T_{2} / T_{1}$ is large. These may correspond to splitting of major peaks in population density observations.
\end{abstract}

\section{Introduction and preliminary results}

Many deterministic mathematical models have been developed in attempts to describe variations in population levels of a single biological species which is isolated from environmental factors and from interaction with other species (see, for example, the books by May [14], Maynard Smith [15] and Pielou [19]). Time lags or delay terms are used to model biological features such as regeneration time and natality lag; or incubation time and infectious period in an epidemic model. Hutchinson [9] suggests a model with a single time lag $T$ :

$$
\frac{d N(t)}{d t}=R N(t)(1-N(t-T) / K),
$$

\footnotetext{
'School of Australian Environmental Studies, Griffith University, Nathan, Queensland 4111.

${ }^{2}$ Department of Mathematics, University of Victoria, Victoria, British Columbia, Canada V8W 2 Y2. (c) Copyright Australian Mathematical Society 1983
} 
where $R, K, T$ are positive constants, to describe the population level $N(t)$ at time $t$. Here $R$ is the intrinsic rate of increase, and $K$ is the carrying capacity. This is a generalised logistic model in which the self-regulatory term has been modified by the inclusion of the delay term. Hutchinson's model has been extensively studied (see, for example, Wright [25], Hale [6], Nussbaum [16] and Kaplan and Yorke [12]) and many of the properties of its solutions are now understood.

However, it is often biologically unrealistic to assume that the self-regulatory term should depend on just one point in past time. Recently, attention has turned to models which incorporate two time delays in the self-regulating process; for example, Cooke and Yorke [4], Hale [7, 8], Nussbaum [17, 18], Silkowski [21], and Stech [22, 23]. One such model is an obvious extension of Hutchinson's to the form

$$
\frac{d N(t)}{d t}=R N(t)\left(1-B N\left(t-T_{1}\right)-C N\left(t-T_{2}\right)\right)
$$

where there are two constant time lags, $T_{2} \geqslant T_{1}>0$, in the self regulating mechanism, and the constants $B$ and $C$ specify the relative importance of the simple intrinsic growth term and the two time delay terms. Observe that (1.2) contains (1.1) as a special case for any of the three conditions $T_{1}=T_{2}, B=0$ or $C=0$. The unique non-trivial equilibrium population $N^{*}=(B+C)^{-1}$ is assumed positive and can be interpreted as a carrying capacity. Kitching [13] has indicated that the life cycle of the Australian blowfly Lucilia cuprina, has three time delay features which need to be considered in modelling its population dynamics. Unfortunately we are unable to analyse such complicated models at this stage, but the inclusion of extra delay terms is an attempt to add realism.

Setting $N(t)=N^{*}(1+\eta(t))$, and nondimensionalising time using the smaller time lag $T_{1}$ (setting $t=T_{1} \tau$ and denoting $d \eta / d \tau \equiv \dot{\eta}$ ) equation (1.2), reduces to the form

$$
\dot{\eta}(\tau)=-(1+\eta(\tau))[f \eta(\tau-1)+g \eta(\tau-\theta)]
$$

where $\theta=T_{2} / T_{1} \geqslant 1, f=B R T_{1} N^{*}$ and $g=C R T_{1} N^{*}$, so that $f+g=R T_{1}>0$.

Equation (1.3) is a differential delay equation and the values of $\eta(\tau)$ for $-\theta \leqslant \tau \leqslant 0$ must be given as initial data before we can attempt to integrate it. We will usually consider $\eta(\tau)=\eta(0)$, a constant, for $-\theta \leqslant \tau \leqslant 0$, as interest lies in the steady state behaviour of the solutions rather than in the transient response to pathological initial data. Following Wright [25, page 67], assume $\eta(0) \neq-1$, then provided that $\eta(s) \neq-1$, for $0 \leqslant s \leqslant \tau,(1.3)$ can be integrated to give

$$
1+\eta(\tau)=(1+\eta(0)) \exp \left\{-f \int_{-1}^{\tau-1} \eta(s) d s-g \int_{-\theta}^{\tau-\theta} \eta(s) d s\right\} .
$$


This equation defines a step by step procedure by which a finite unique value of $\eta(\tau)$ is obtained. The following results are now readily proved:

\section{THEOREM 1.}

(a) Corresponding to every bounded integrable set of values $\eta(\tau)$ for $-\theta \leqslant \tau \leqslant 0$, there is a unique solution of (1.3) for $\tau>0$.

(b) For $j+g \neq \overline{0}$, as $\tau \rightarrow \infty$, the only possible constant limits of $\eta(\tau)$ are 0 and -1 . For $f+g=0$, the limit is not determined by this method.

(c) For all $\tau>0, \eta(\tau) \gtreqless-1$, according as $\eta(0) \gtreqless-1$.

(d) For $\eta(0)<-1$, and if $f>0, g>0$, then $\eta(\tau) \rightarrow \infty$, monotonically as $\tau \rightarrow \infty$.

(e) For $\eta(0)>-1$, and if the number of zeros of $\eta(\tau)$ is bounded, then $\eta(\tau) \rightarrow 0$ as $\tau \rightarrow \infty$.

(f) For $\eta(0)>-1, f>0, g>0$ and if the number of zeros of $\eta(\tau)$ is unbounded, then

$$
-1<\eta(\tau)<e^{\theta(f+g)}-1 .
$$

(g) For $\eta(0)>-1, f>0, g>0$ and $f+g>1$, then the zeros of $\eta(\tau)$ are unbounded.

(h) For $f>0$, and $g>0, \eta(\tau) \rightarrow 0$ as $\tau \rightarrow \infty$, provided that $0<\alpha \leqslant 1$, where

$$
\alpha=\theta(f+g) \text {. }
$$

The proof of Theorem 1 is given in Appendix A. The above results are extensions to two time delay equations of similar results obtained by Wright [25] for a one time delay equation, i.e. Hutchinson's equation. In (h) above, the range of values of $\alpha$ is not limiting. At the expense of great detail, Wright [25] extends the range of values of $\alpha$ for which $\eta(\tau) \rightarrow 0$ as $\tau \rightarrow \infty$, to $0<\alpha \leqslant 1.5$, and conjectures that the upper limit is $\alpha<\pi / 2 \approx 1.5708$. This conjecture is supported by numerical calculations of the solutions of (1.1) (Jones [10], Braddock and van den Driessche [2]). Again, a similar conjecture can be made regarding the limiting behaviour of the solutions of (1.3), with the definition $\alpha=\theta(f+q)$, and is supported by numerical results.

The linearised differential-difference equation associated with (1.3) is

$$
\dot{\eta}(\tau)=-f \eta(\tau-1)-g \eta(\tau-\theta),
$$

which, on seeking solutions of the form $\eta(\tau) \propto \exp (\lambda \tau)$, yields the eigenvalue equation

$$
\lambda=-f e^{-\lambda}-g e^{-\lambda \theta} .
$$

By standard theory the equilibrium point $N^{*}$ of the nonlinear equation is locally stable to small perturbations if the solutions to (1.5) exhibit either 
exponential or damped oscillatory decay: equivalently, all of the complex eigenvalues of (1.6) have negative real parts. Note that the complex roots of the quasi-polynomial equation (1.6) occur as conjugate pairs; thus we restrict attention to solutions $\lambda=\alpha+i \beta$ with $\beta \geqslant 0$. Recently, interest has been focused on the bifurcation of solutions as the eigenvalue $\lambda$ passes into the right half plane; see Hale $[6,7,8]$, Nussbaum $[16,17,18]$, Stech $[22,23]$.

A special case of (1.3), with $f=-g$, has been considered by Cooke and Yorke [4] in the mathematical modelling of sexually transmitted diseases. Their work describes the various stability properties of (1.3) for $f+g=0$, and they also obtain some limit cycle solutions. The condition $f+g>0$ has greater biological significance with respect to the above model and, in fact, we will show that their line $(f+g=0)$ separates two completely different forms of eigenvalues of (1.6), in the $(f, g)$ plane. Stech $[22,23]$ considers the special case $f+g=1, \theta=3$, and derives conditions for linear stability and also discusses the stable and unstable bifurcations of the solutions. Nussbaum $[17,18]$ considers (1.6) for $1<\theta<2$ and for the special case $\theta=3$, and derives conditions for the linear stability of a more general model equation. His work on the eigen-equation (1.6) is relevant to this discussion.

The remainder of this paper considers in detail the properties of the eigenvalues of (1.6). These properties govern the linear stability properties of (1.3) and also the existence of the limit cycle solutions. The special case $f=g$ is discussed in detail.

2. The real and pure imaginary roots and the linear stability region for $f=g$

Consider (1.3) with $f=g$, so that

$$
\dot{\eta}(\tau)=-f(1+\eta(\tau))(\eta(\tau-1)+\eta(\tau-\theta)) .
$$

The linearised form of this equation is

$$
\dot{\eta}(\tau)=-f(\eta(\tau-1)+\eta(\tau-\theta)),
$$

with eigenvalue equation

$$
\lambda=-f H(\lambda, \gamma, \theta), \quad \text { where } H(\lambda, \gamma, \theta)=e^{-\lambda}+\gamma e^{-\lambda \theta},
$$

and $\gamma=1$. The general function $H(\lambda, \gamma, \theta)$ is defined at this point although only the particular parameter value $\gamma=1$ is required here: the more general form will be used at a later stage. Let $\lambda=\alpha(f, \theta)+i \beta(f, \theta)$ be the complex eigenvalues satisfying (2.3), with $\beta \geqslant 0$. The region of linear stability is the set of all $(f, \theta)$, with $\theta>1$, such that $\alpha<0$ for all solutions of (2.3). Before describing this region, we discuss the purely real roots $\alpha=r, \beta=0$; and the purely imaginary roots $\alpha=0, \beta=d$, of (2.3). 


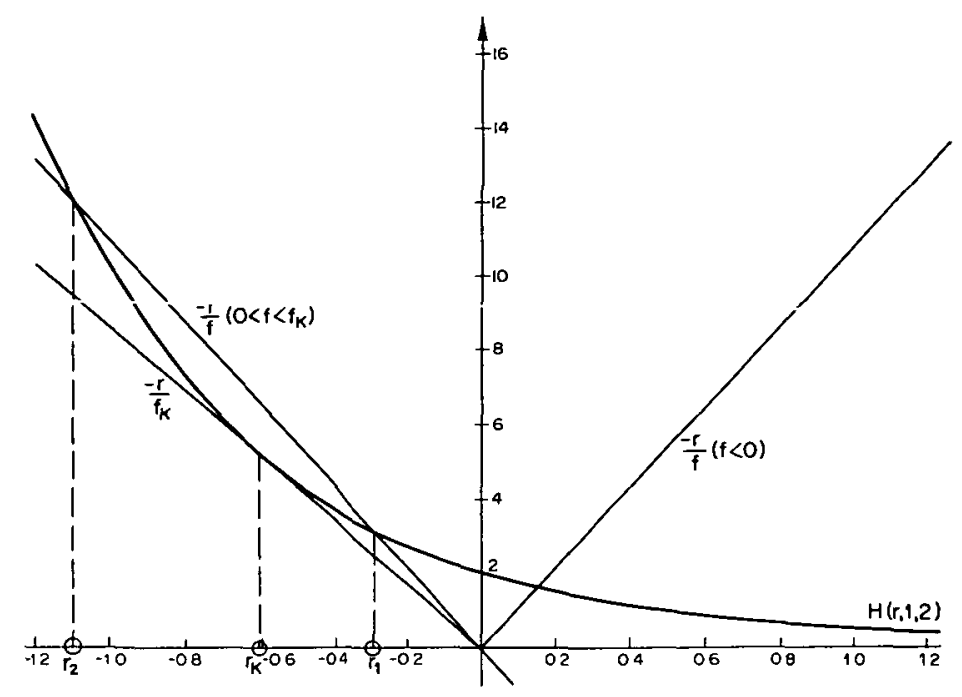

Figure l. Graph of $H(r, 1,2)$ defined in (3.3) showing the single positive intersection for $f<0$, the double root for $f=f_{K}$, and the two negative roots for $0<f<f_{K}$.

The real roots, which yield exponential solutions of (2.2), satisfy

$$
-r / f=H(r, 1, \theta)=e^{-r}+e^{-r \theta} .
$$

The function $H(r, 1, \theta)$ is positive for all $r$ and $\theta$, and is a monotonically decreasing function of $r$ when the parameter $\theta>1$. Figure 1 illustrates the behaviour of $H(r, 1, \theta)$ for $\theta=2$, and the same quantitative behaviour holds for all $\theta>1$. The intersections of $H(r, 1, \theta)$ with the straight line $-r / f$, give the purely real eigenvalues $\alpha=r$; these solutions may be summarized as follows:

\section{THEOREM 2.}

(a) If $f<0$, then the straight line $-r / f$ has a positive gradient and there is a single real positive root of (2.3). Equation (2.1) is then unstable to small oscillations.

(b) If $f=0$, then (2.3) is trivially satisfied by $r=0$.

(c) If $f>0$, then the gradient of the straight line is negative, and there are three possibilities:

(i) There is a double real negative root $r_{K}$ corresponding to the critical values $f=f_{K}$. At $f=f_{K}$, the straight line is tangent to the function $H(r, 1, \theta)$ (see below).

(ii) If $0<f<f_{K}$, then there are two distinct real negative roots $r_{1}$ and $r_{2}$ such that $r_{2}<r_{K}<r_{1}<0$.

(iii) If $f>f_{K}$, then there are no purely real roots. 
Remarks. The proof of Theorem 2 is essentially graphical and obvious from Figure 1. Case (c) proves the existence of the critical value $f_{K}$ such that there is a double negative root $r_{K}$. At this point, the straight line (which passes through the origin) is tangent to $H(r, 1, \theta)$, so that

$$
\left.\frac{d H}{d r}(r, 1, \theta)\right|_{r_{K}}=\frac{H\left(r_{K}, 1, \theta\right)}{r_{K}}
$$

and hence

$$
e^{-r_{K}}\left(1+r_{K}\right)+e^{-r_{K} \theta}\left(1+\theta r_{K}\right)=0 .
$$

Further

$$
-1 / f_{K}=\left.\frac{d H}{d r}(r, 1, \theta)\right|_{r_{K}},
$$

since the gradient of the straight line is $-1 / f$. Note that for $\theta=1$, then $r_{K}=-1$ and $2 f_{K}=e^{-1}$, which is in agreement with Wright's [25] results for the generalised logistic equation. For large $\theta, f_{K} \approx c \theta^{-1}, r_{K} \approx-f_{K}-\theta^{-1}$, where $c e^{c+1}=1$. Numerical values of $r_{K}$ and $f_{K}$ for various $\theta$ are readily obtained by applying Newton's method to (2.5), and the results are contained in Table 1.

TABLE 1 . Values of critical $f\left(f_{K}\right)$ corresponding to a double negative real root $r\left(r_{K}\right)$ for various values of $\theta$.

\begin{tabular}{ccc}
\hline$\theta$ & $r_{K}$ & $f_{K}$ \\
\hline 1.2 & -0.902 & 0.167 \\
1.4 & -0.812 & 0.151 \\
1.6 & -0.732 & 0.138 \\
1.8 & -0.665 & 0.127 \\
2.0 & -0.607 & 0.117 \\
3.0 & -0.418 & 0.083 \\
4.0 & -0.316 & 0.064 \\
5.0 & -0.254 & 0.052 \\
6.0 & -0.212 & 0.044 \\
7.0 & -0.182 & 0.038
\end{tabular}

The purely imaginary roots of (2.3) yield neutral solutions of (2.2), which are not exponential but purely oscillatory in character. Setting $\lambda=i d, d \geqslant 0$, and extracting real and imaginary parts from (2.3), gives

$$
\begin{gathered}
0=\cos d+\cos d \theta=2 \cos \frac{d}{2}(\theta-1) \cos \frac{d}{2}(\theta+1), \\
d=f(\sin d+\sin d \theta) .
\end{gathered}
$$

Equation (2.7) is satisfied by

$$
\begin{aligned}
d_{n}(\theta) & =\pi(2 n+1) /(\theta+1), \quad n \in J, \quad \text { and } \\
d^{m}(\theta) & =\pi(2 m+1) /(\theta-1), \quad m \in J,
\end{aligned}
$$


where $J$ is the set of non-negative integers. (The roots $d^{m}(\theta)$ cannot satisfy (2.8) but they are required in the stability proof.) The roots $d_{n}(\theta)$ satisfy $(2.8)$ provided that $f=f_{n}(\theta), n \in J$, where

$$
f_{n}(\theta)=d_{n}(\theta) / 2 \sin d_{n}(\theta) .
$$

Since the equation is unstable for $f<0$, those roots $d_{n}(\theta)$ for which $\sin d_{n}(\theta)<0$ are not required in the following discussion.

Now $d_{c}(\dot{\theta})=d_{0}(\tilde{\theta})$ is the smallest positive neutral root and $d_{c}(\dot{\theta})=\pi /(\dot{\theta}+1)$ $<\pi / 2$ for $\theta>1$. The corresponding value of $f$ is

$$
f_{c}(\theta)=\pi /(2(\theta+1) \sin (\pi /(\theta+1))) .
$$

Since the function $F(y)=y /(2 \sin y)$ is monotonically increasing for $0 \leqslant y \leqslant$ $\pi / 2, F(y)$ is bounded by $1 / 2 \leqslant F(y) \leqslant \pi / 4$; obviously $1 / 2 \leqslant f_{c}(\theta)<\pi / 4$, for $\theta>1$. Further, for $d_{n}(\theta) \geqslant \pi / 2,\left|f_{n}(\theta)\right| \geqslant \frac{1}{2}\left|d_{n}(\theta)\right| \geqslant \pi / 4$. It then follows that $f_{c}(\theta)=f_{0}(\theta)$ is the smallest positive value of $f$ for which a neutral root exists. These purely imaginary roots are also characterized in a qualitative way by Claeyssen [3].

Before considering the stability of the equation, we obtain a result regarding the distribution of the roots. For a complex eigenvalue $\lambda=\alpha+i \beta, \beta \geqslant 0$, the real and imaginary parts of (2.3) give

$$
\begin{gathered}
\alpha=-f\left(e^{-\alpha} \cos \beta+e^{-\alpha \theta} \cos \beta \theta\right)=-f \cos \beta H(\alpha, \gamma, \theta), \\
\beta=f\left(e^{-\alpha} \sin \beta+e^{-\alpha \theta} \sin \beta \theta\right),
\end{gathered}
$$

where $H(\alpha, \gamma, \theta)$ is defined in (2.3) and $\gamma=\cos \beta \theta / \cos \beta$. If $\alpha \geqslant 0$ and $f>0$, then the right-hand side of (2.11) is bounded by $f\left(e^{-\alpha}+e^{-\alpha \theta}\right)$, and thus for $\theta>1, \beta \leqslant 2 f$. Similarly it can be shown that $\alpha \leqslant 2 f$ if $\alpha \geqslant 0$. Thus the unstable eigenvalues $(\alpha \geqslant 0)$ are bounded by the box $0 \leqslant \alpha \leqslant 2 f, 0 \leqslant \beta \leqslant 2 f$ for all $\theta>1$ and $f>0$.

The critical value $f_{c}(\theta)$, defined by (2.9), delineates the linear stability region; the results are summarised as follows:

\section{THEOREM 3.}

(a) If $0<f<f_{c}(\theta)$, then all eigenvalues $\lambda=\alpha+i \beta$ have $\alpha<0$.

(b) If $f \geqslant f_{c}(\theta)$, then there is at least one eigenvalue with $\alpha \geqslant 0$.

The proof of this theorem involves tedious enumeration of the imaginary roots and is given in Appendix B. This theorem indicates that one of the eigenvalues bifurcates, passes from the left-half plane into the right-half plane, as the parameter $f$ increases in value through $f_{c}(\theta)$. The critical value is defined in this way, i.e. see (2.9), purely for convenience; obviously a bifurcation is achieved by varying either $f$ or $\theta$, or both together, so as to cross the critical line $f_{c}(\theta)$ in the 
$(\theta, f)$ plane. As indicated in the proof (see Appendix B), other eigenvalues bifurcate as $f$ increases in value past $f_{1}, f_{2}, \ldots$. These may be termed secondary bifurcations.

\section{The limit cycle solutions for $f=g$}

The above results are similar in many respects to the properties of Hutchinson's equation [9],

$$
\dot{\eta}(t)=-\hat{\alpha} \eta(t-1)(1+\eta(t)),
$$

(for $0<\hat{\alpha} \leqslant \alpha_{c}=\pi / 2$ ) which has attracted considerable attention (see, for example, Wright [25], Jones [10] and Hale [6]). Note that for $\theta=1,(2.1)$ reduces to the above form with $\hat{\alpha}=2 f$. However, the discussion of the linear stability region for (2.1) has been complicated because of the presence of the second time lag term for $\theta>1$.

In discussing the properties of the solutions of (2.1) for $f \geqslant f_{c}(\theta)$, it is appropriate first to consider the properties of the solutions of (3.1) for $\alpha \geqslant \alpha_{c}=\pi / 2$. It is well known that

(a) for $\hat{\alpha}=\alpha_{c}=\pi / 2$, the linear equation corresponding to (3.1), has pure imaginary eigenvalues $\lambda_{0}= \pm i \pi / 2$, and that the solution of (3.1) has period of four units (Jones [10]).

(b) As $\hat{\alpha}$ increases through $\pi / 2$, the real part of the dominant eigenvalue $\lambda_{0}$, changes sign from negative to positive, and the solution bifurcates.

Wright [25] shows that for $\hat{\alpha}>\pi / 2$, the solutions of (3.1) are oscillatory; Jones [10] mathematically proves the existence of these solutions and determines the period as being greater than 4 units. Further, Kaplan and Yorke [12] prove a stability result for the limit cycle solutions for a general class of delay equations which includes (3.1).

The limit cycle solutions of (3.1) have been studied by Jones [10] and Braddock and van den Driessche [2], over a large range of parameter values. As $\hat{\alpha}$ increases, both the period and amplitude of the oscillatory solutions increase rapidly, although the solutions are restricted by $\eta(t)>-1$ (compare with Theorem 1, part (f)). Biological reasons restrict the parameter range for $\hat{\alpha}$ to $0<\hat{\alpha} \lesssim 2.5$, although Jones [10] and Braddock and van den Driessche [2] have obtained solutions for $\hat{\alpha} \leqslant 4$.5. Braddock and van den Driessche [2] discuss the numerical difficulties associated with the solution of (3.1) using large values of $\hat{\alpha}$. They also show that the change of variable

$$
\zeta(\tau)=\ln (1+\eta(\tau))
$$

transforms (3.1) to the form

$$
\dot{\zeta}(\tau)=\hat{\alpha}(1-\exp [\zeta(\tau-1)]),
$$


and that solutions can be obtained for an extended range of values of $\hat{\alpha}$. Using (3.3), the authors have recently calculated numerical solutions for $\hat{\alpha}$ near $5 \pi / 2 \approx$ 7.854. At $\hat{\alpha}=5 \pi / 2$, a second eigen pair cross the imaginary axis into the right-hand half plane but there is no discernible change in the nature of the numerical solutions. Such a process will be called a secondary bifurcation and will be shown to have a dramatic effect in the two time lag model.

Applying the change of variable (3.2) to the two time lag model (2.1) yields

$$
\dot{\zeta}(\tau)=2 f-f\{\exp [\zeta(\tau-1)]+\exp [\zeta(\tau-\theta)]\},
$$

which is readily solved numerically using the techniques described by Braddock and van den Driessche [2]. Numerical solutions were obtained for $\theta=1.5,2.5,3.5$ and for all integer values $\theta=2,3,4, \ldots, 11$, as well as for a wide range of values of the parameter $f$. The tested values of $f$ were not uniformly distributed since the behaviour of the solutions near $f_{n}=f_{n}(\theta)$, is of particular interest.

The numerical results reinforce the stability analysis in that:

(a) for $0<f \leqslant f_{K}(\theta)$, the solutions exhibit exponential decay, with some more rapidly decaying oscillations also present, these being due to other subdominant eigenvalues;

(b) for $f_{K}(\theta)<f<f_{c}(\theta)$, the solutions exhibit oscillatory decay;

(c) at $f=f_{c}(\theta)$, the solutions persist in the form of a neutral oscillation with a period of $2 \pi / d_{0}(\theta)=2(\theta+1)$ (see above).

The rate of decay of the solutions for $0<f<f_{c}(\theta)$, depends heavily on the value of $\theta$; and, as $\theta$ increases, the decay rate decreases. This agrees quantitatively with the figures in Table 1 , in which the magnitude of the double exponential root, $\left|\alpha_{K}\right|$, decreases as $\theta$ increases. (Note that $\alpha_{K}$ is not a measure of the decay rate for other values of $f \neq f_{K}$, but it gives a quantitative explanation.)

The behaviour of the solutions for $f>f_{c}(\theta)$ is much more exciting, for they appear to bifurcate to produce stable limit cycles, and then exhibit a further strange behaviour which we called secondary bifurcation. The behaviour of the limit cycles for $f>f_{c}(\theta)$ is well illustrated by considering the case $\theta=10$. The neutral roots are then $d_{n}=\pi(2 n+1) / 11$, and the corresponding critical values of $f$ are $f_{c}=f_{0}=0.506, f_{1}=0.567, f_{2}=0.721, f_{3}=1.097$. Due to the previously described numerical difficulties, the solution could not be extended to the value $f_{3}$. For $f_{0}<f<f_{1}$, the limit cycle (in the phase plane), see Figure 2 , is very similar to those obtained by Jones [10] for Hutchinson's equation. However, a cusp is apparent just after the maximum value of $\eta(\tau)$ is attained; the cusp becomes more marked as $f$ increases toward $f_{1}$. As the value of $f$ increases through $f_{1}$, the cusp develops into a loop as shown in Figure 3; a much smaller loop also develops near the minimum value of $\eta(\tau)$. This picture remains until $f$ increases in value to approximately $f_{2}=0.721$ where a second cusp develops inside the loop 


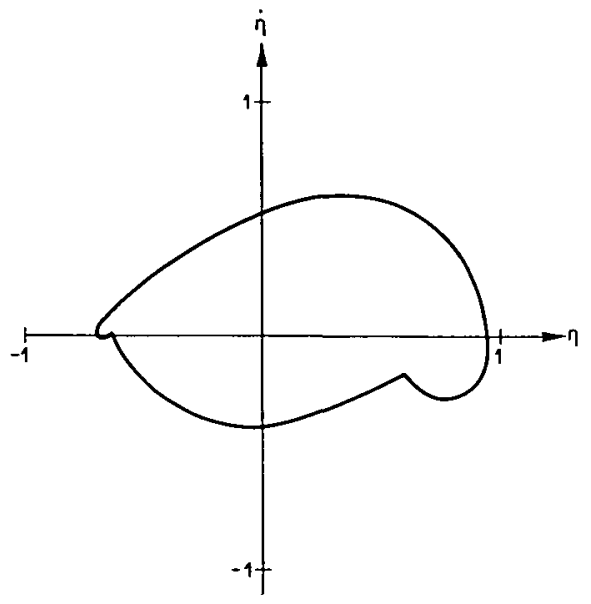

Figure 2. Limit cycle in the phase plane for (3.1), with $\theta=10, f=g=0.54$.

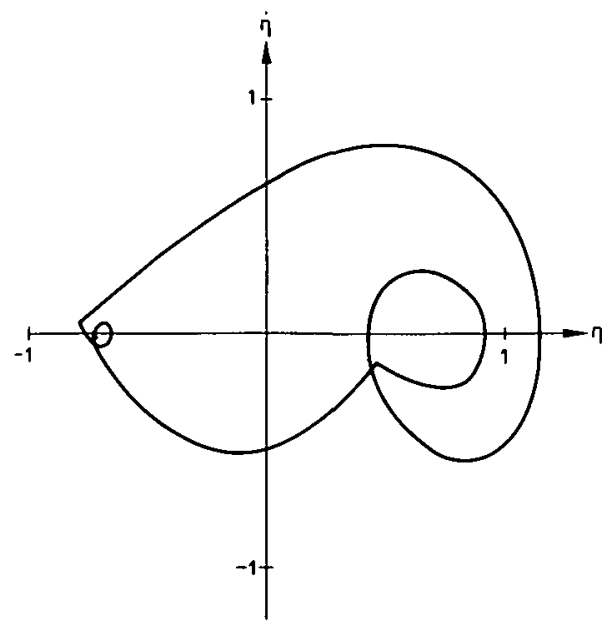

Figure 3. Limit cycle in the phase plane for (3.1), with $\theta=10, f=g=0.60$. 


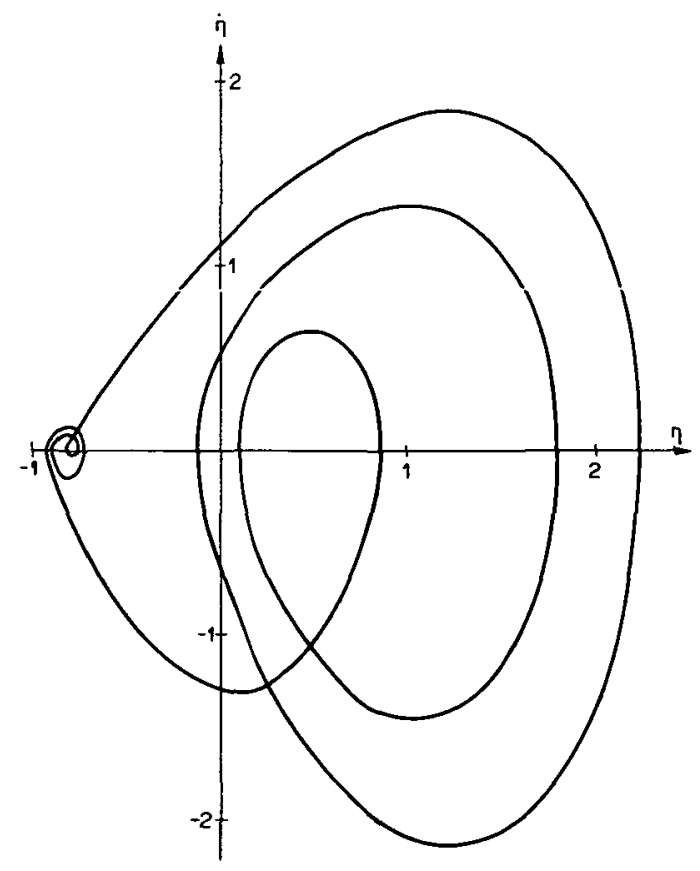

Figure 4. Limit cycle in the phase plane for (3.1), with $\theta=10, f=g=0.74$.

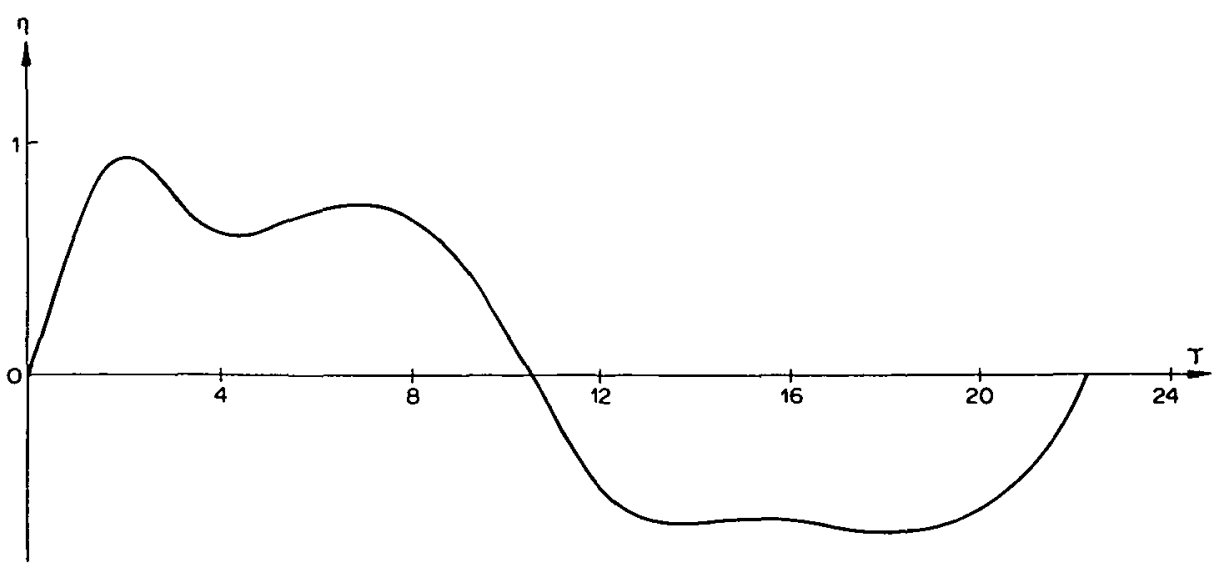

Figure 5. Population curve $\eta=\eta(\tau)$, showing the limit cycle for (3.1), with $\theta=10, f=g=0.54$. 


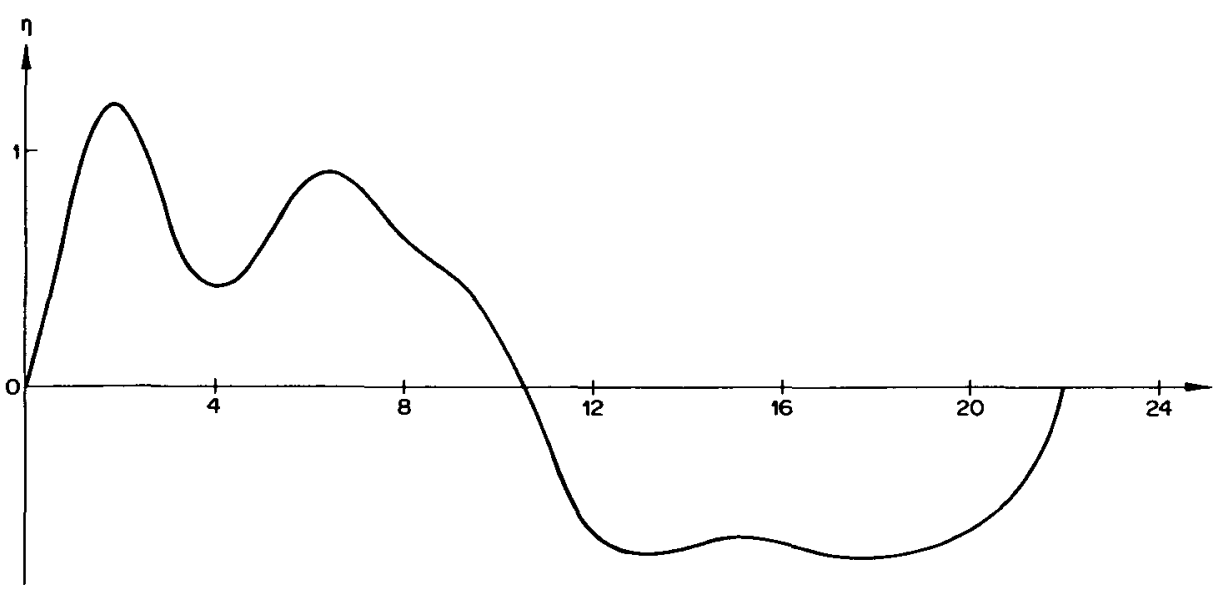

Figure 6. Population curve $\eta=\eta(\tau)$, showing the limit cycle for (3.1), with $\theta=10, f=g=0.60$.

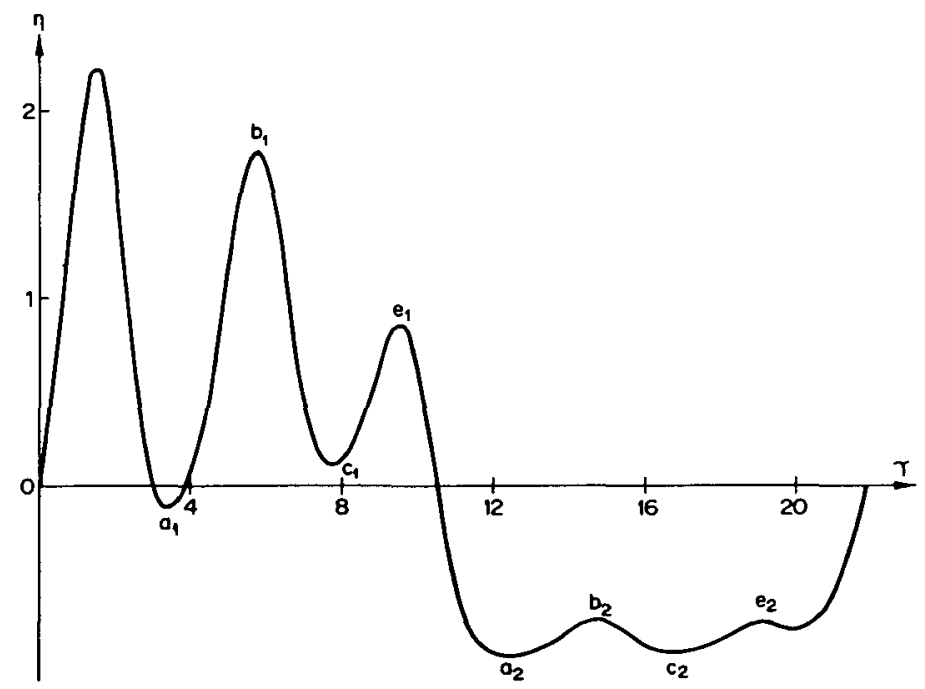

Figure 7. Population curve $\eta=\eta(\tau)$, showing the limit cycle for (3.1), with $\theta=10, f=g=0.74$.

(which has increased in size). For $f>f_{2}$, a second major loop develops for $\eta(\tau)$ near its maximum together with another secondary loop near the minimum (see Figure 4). The corresponding population graphs i.e. graphs of $\eta$ as a function of $\tau$, show that the major peak splits into two and then into three, and that small peaks develop in the troughs (see Figures 5, 6 and 7). 
In determining the time taken to transverse the different sections of the limit cycle, consider the maximum and minimum points $a_{1}, a_{2}, b_{1}, b_{2}, c_{1}, c_{2}$, etc. as defined in the $\tau, \eta$ plane in Figure 7. Let $\tau_{e}=$ time at point $e$, then it is found that

$$
\begin{array}{ll}
\tau_{a_{2}}-\tau_{a_{1}}=9.00 \pm 0.01, & \tau_{b_{2}}-\tau_{b_{1}}=9.00 \pm 0.01, \\
\tau_{c_{2}}-\tau_{c_{1}}=9.00 \pm 0.01, & \tau_{e_{2}}-\tau_{e_{1}}=9.31 \pm 0.01 .
\end{array}
$$

The last valuie differs fromin the value $\theta-1=9$ presumabily since the small peak $e_{2}$ is not yet fully developed. Further, it is also found that the time from peak to peak (or trough to trough), i.e. $\tau_{c_{1}}-\tau_{a_{1}}$, etc. is between 4.0 and 4.6.

Similar results to the above are found for all tested $\theta \geqslant 4$. For $\theta<4, f_{1}>1.0$ and the numerical solution could not be extended far enough with sufficient accuracy to determine if additional loops form inside the limit cycle. For $\theta \geqslant 4$, additional loops form as the value of $f$ passes through the values $f_{n}, n \geqslant 1$, and in each case, the time difference between the corresponding large and small peaks is approximately $\theta-1$ units.

Near its bifurcation point, i.e., $f \approx f_{c}(\theta)$, the numerical solutions to (3.1) show a period near $2(\theta+1)$, and for large values of $\theta$, this period is much greater than 4 . For $\theta=1$, this gives a period of 4 units, corresponding to the period of the bifurcating solution for Hutchinson's model. After the solution passes its first major peak (see Figure 2), $\eta(\tau)$ falls rapidly. Now (2.1) contains two similar expressions, i.e., $f(1+\eta(\tau)) \eta(\tau-\theta)$ and $f(1+\eta(\tau)) \eta(\tau-1)$. Because the peaks in $\eta(\tau)$ are relatively narrow compared to the time $\operatorname{lag} \theta$, the delay term in the expression $f(1+\eta(\tau)) \eta(\tau-\theta)$ cannot 'feel' the influence of the peak. However the delay expression $f(1+\eta(\tau)) \eta(\tau-1)$ (which, near bifurcation, has a period of 4) acts to produce the second major peak. If $\theta$ is large enough, this term will act to produce further peaks. However, the larger delay term finally encounters the first major peak and $\eta(\tau)$ becomes small. The secondary peaks, at a time lag of $\theta-1$ behind the corresponding major peaks, are produced by this large time lag, although the nonlinear nature of equation (2.1) greatly reduces the amplitude.

\section{Some results for general $f$ and $g$}

We return to the general two time lag problem equation (1.3) for unrestricted values of the parameters $f$ and $g$, and the problem of determining the eigenvalues $\lambda(f, g, \theta)$ and the zeros of the real and imaginary parts. It is convenient first to consider the purely real roots $\alpha=r$ and $\beta=0$ of (1.6), and on substituting we have, for $f \neq 0$,

$$
-r / f=H(r, \gamma, \theta)
$$


where $\gamma=g / f$, and $H(r, \gamma, \theta)$ is defined in (2.3). The roots of (4.1) are described by the intersections of the straight line $r / f$ and the function $H(r, \gamma, \theta)$ (see Theorem 2), but here the situation is complicated by the behaviour of $H(r, \gamma, \theta)$. The values of $\lim _{r \rightarrow-\infty} H(r, \gamma, \theta)$, and $H(0, \gamma, \theta)$ are such that several tangency conditions must be considered (see (2.4)). The root properties are discussed in detail in Appendix $\mathrm{C}$, together with the use of the tangency conditions in calculating the corresponding curves in the $(f, g)$ plane (see Figure 8).

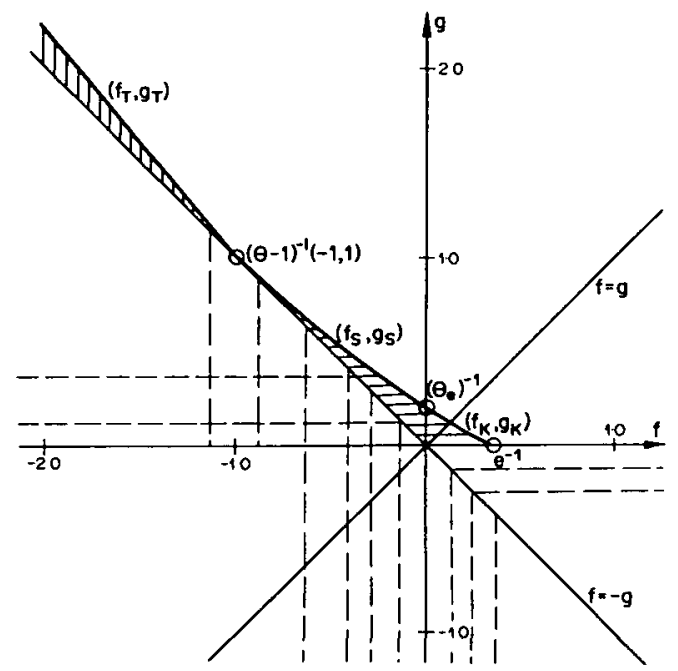

Figure 8. The real roots of equation (1.5). The location of the double real positive roots $\left(f_{T}, g_{T}\right)$, and the double real negative roots $\left(f_{S}, g_{S}\right)$ and $\left(f_{K}, g_{K}\right)$ are shown for $\theta=2$. Broken horizontal lines, solid horizontal lines, broken vertical lines, and solid vertical lines indicate regions with one negative, two negative, one positive and two positive roots, respectively.

The implications of the discussion in Appendix $\mathrm{C}$ are that positive real eigenvalues exist in large areas of the $(f, g)$ plane, and (1.3) is linearly unstable on these areas. These results are summarised as Theorem 4.

THEOREM 4. Equation (1.3) is linearly unstable for the following parameter values:

(a) $f<0, g \leqslant 0$, all $\theta$ (see Appendix C.1, part (a)).

(b) $f<0, g>0, f+g<0$, all $\theta$ (see Appendix C.2, part (b)).

(c) $f+g=0, f<-(\theta-1)^{-1}$ (see Appendix C.3, part (b)(iii)).

(d) $f>0, g<0, f+g<0$, all $\theta$ (see Appendix C.4, part (a)).

(e) The region bounded by $f+g=0$, and the curve $\left(f_{T}, g_{T}\right)$ (see Appendix C. 4 part (b), and the remarks in (C.5)). 
The proof of each part of the theorem is contained in the appropriate part of Appendix $\mathrm{C}$ noted above. The curve $\left(f_{T}, g_{T}\right)$ is obtained by applying the Newton technique to the appropriate tangency condition (see equation (C.1)). A corollary to the above theorem is that the remainder of the $(f, g)$ plane contains only negative real roots for all $\theta>1$.

We now consider the purely imaginary eigenvalues $\alpha=0, \beta=d \geqslant 0$, of (1.6), which correspond to neutral oscillations of (1.3). Such eigenvalues must satisfy both equations

$$
f \cos d+g \cos d \theta=0,
$$

and

$$
d=f \sin d+g \sin d \theta .
$$

Here we are interested in the curves in the $(f, g)$ plane which correspond to neutral roots; it is preferable to regard $d$ as an independent variable and seek $f=f(d, \theta)$ and $g=g(d, \theta)$, treating $\theta$ as a parameter. Solving (4.2) and (4.3), provided $\sin d(\theta-1) \neq 0$, yields

$$
\begin{array}{cc}
f=-\frac{d \cos d \theta}{\sin d(\theta-1)}, & \text { provided } \cos d \theta \neq 0, \\
g=\frac{d \cos d}{\sin d(\theta-1)}, & \text { provided } \cos d \neq 0 .
\end{array}
$$

Note that both $f$ and $g$ are even functions of $d$, and that $\lim _{d \rightarrow 0} g=\lim _{d \rightarrow 0} f=$ $(\theta-1)^{-1}$, the limit of the stable region of the line $f+g=0$, as described by Cooke and Yorke [4].

We turn briefly to the special cases which are excluded in the derivation of (4.4). We consider only the details of the case of $\cos d=0$, since the other case is very similar. If $\cos d=0$, then from (4.2) we have the trivial case $g=0$, or we must also have $\cos d \theta=0$. The case $g=0$ gives $d=\pi(2 n+1) / 2, f=$ $(-1)^{n} \pi(2 n+1) / 2, n \in J$; as expected from Wright's results. However, if both $\cos d=0$ and $\cos d \theta=0$, then these two conditions imply that $\theta=2 n+1$, $n \in J$. (4.3) yields families of straight lines with gradients \pm 1 , and $f$ intercepts $\pm \pi(2 n+1) / 2, n \in J$. A final special case arises if the linear system (4.2) and (4.3), for $f$ and $g$ as unknowns, is singular, i.e., if $\sin d(\theta-1)=0$. This implies that $d=n \pi /(\theta-1), n \in J$, and then (4.2) implies that $f \pm g=0$. Thus the cases $f+g=0$ (see Cooke and Yorke [4]) and $f-g=0$ are singular examples of the general two time lag equations. This linear dependence between the two equations greatly simplifies the identification of the neutral roots and the pursuance of the stability proof (see Appendix B).

Stech [22, 23] and also Hale [8] consider a special form of (1.3) with $\theta=3$, $f \geqslant 0$ and $g=1-f$. Hale shows stability for this case, for $g$ between zero and a 
number "just larger than $\frac{1}{4}$ ". In the above case, the system (4.4) reduces to

$$
f=-\frac{d \cos (3 d)}{\sin (2 d)}, \quad 1-f=\frac{d \cos (d)}{\sin (2 d)},
$$

in which we seek solutions for both $f$ and $d$. Eliminating $f$ leads to the equation

$$
\sin 2 d=d(\cos d-\cos 3 d) \text {, or } d=(2 \sin d)^{-1} \text {. }
$$

Graphically it is obvious that there are an infinite number of solutions to the equation $\sin (d)=(2 d)^{-1}$, and that these solutions are approximated by:

$$
d_{\rho}^{1} \approx(2 \rho+1) \pi-[2(2 \rho+1) \pi]^{-1} \cdots,
$$

and

$$
d_{\rho}^{2} \approx 2 \rho \pi+(2(2 \rho \pi))^{-1} \cdots,
$$

for large $\rho$. Further, the corresponding values of $f$ are all negative. The first positive root of (4.6) is readily seen to be near $\pi / 4=0.785$ radians. Direct iteration applied to (4.6) yields the root $d^{*}=0.740841$ radians (correct to 6 significant figures); the corresponding value of $f$ is $f^{*}=0.736359$. We thus conjecture that the equation considered by Stech $[22,23]$ and also Hale [8], is linearly stable for $0 \leqslant g=1-f<0.263641$, and that the solution bifurcates as $g$ passes through this upper limit.

In general, the results of our numerical solutions of (1.3) for selected values of $f, g$ and $\theta$, suggest that there is a zone of linear stability for small $f$ and $g$, and that the curves (4.4) play a major part in delimiting this region. Unfortunately we have been unable to order the eigenvalues for the general case or to develop a full stability analysis.

However, we have obtained some preliminary results for the linear stability of (1.3), albeit under rather restrictive conditions. First, we return to the general eigen equation (1.6) and set $\lambda=\alpha+i \beta$, to obtain

$$
\begin{aligned}
& \alpha=-f e^{-\alpha} \cos (\beta)-g \mathrm{e}^{-\alpha \theta} \cos (\theta \beta), \\
& \beta=f e^{-\alpha} \sin (\beta)+g \mathrm{e}^{-\alpha \theta} \sin (\theta \beta),
\end{aligned}
$$

on extracting real and imaginary parts. For $\beta>0,(4.8)$ yields

$$
1=f e^{-\alpha}(\sin \beta / \beta)+\theta g e^{-\alpha \theta}(\sin (\beta \theta) / \beta \theta),
$$

the right-hand side of which is bounded above by $|f|+\theta|g|$, for all $\alpha \geqslant 0$ and $\beta>0$. Thus it is not possible to find complex eigenvalues with $\alpha \geqslant 0$, provided that

$$
|f|+\theta|g|<1,
$$


this condition defines a rhombus with vertices $( \pm 1,0)$ and $\left(0, \pm \theta^{-1}\right)$ in the $(f, g)$ plane. When this is combined with the results of Theorem 4 , we have the result:

THEOREM 5. All the zeros of (1.6) have negative real parts, and hence (1.3) is linearly stable, provided that $f, g$, and $\theta>1$ satisfy (4.9) and $f+g \geqslant 0$.

Noiice that the rhombus, for $\hat{j}<\hat{0}$, intersects $\hat{f}+g=\hat{0}$, at the point $(\theta+1)^{-1}(-1,1)$ that $(\theta+1)^{-1}<(\theta-1)^{-1}$, and hence the point $(\theta-1)^{-1}(-1,1)$ lies outside of the rhombus (see Theorem 4 , part (c)).

The derivation of (4.9) depends on only one of the eigen equations, i.e. (4.8), and hence provides only a first approximation to the actual stability region. A more detailed investigation would need to consider the bifurcation of the solutions as the eigenvalues cross into the right-hand half plane. Finally, note that for $\alpha \geqslant 0,(4.7)$ and (4.8) provide bounds of the form

$$
\alpha \leqslant|f|+|g|, \quad \beta \leqslant|f|+|g| .
$$

All roots with $\beta>|f|+|g|$ correspond to $\alpha<0$. Thus in checking for stability, the region to be considered is bounded by (4.10).

\section{Discussion}

The particular two time lag equation (1.3) with $f+g=0$, studied by Cooke and Yorke [4] and Hale [7], is one of the few two lag equations considered in the literature (see also Hale [8], Stech [22, 23], Nussbaum [17, 18]). This investigation of (1.3) for general $f$ and $g$, reveals many fascinating properties of the solutions and suggests that many interesting problems on differential-difference models await further study. In particular, the results in Section 4 and especially Figure 8 for the associated linear equation, show that $f+g=0$ is truly a very special line in the $(f, g)$ plane. It bounds an unstable region on one side $f+g<0$, and the other side contains regions of stability, neutral stability, and instability, and also regions which have not yet been fully investigated. Certain regions of stability are defined in Section 4, in particular the rhombus condition, although more precise estimates are possible for more limited regions. In addition, when $f+g=0$, the nonlinear equation admits $\eta(\tau)$ equal to any constant as a solution. Finally, the point $(\theta-1)^{-1}(-1,1)$ is of particular interest, being the limit point of the neutral curve, the curve on which there is a double negative real root, and the curve on which there is a double positive real root.

The particular case $f-g=0$, considered in Sections 2 and 3, is also of special interest in the two time lag problems. On this line the stability question is 
completely answered, although there remains some doubt regarding the outer limits of the stability region on either side of this line. As described in Section 4, both $f \pm g=0$ are neutral curves when $\theta$ is an odd integer.

Considerations of the general two time lag problem answers some questions regarding the stability (or instability) of equation (1.3), but does not set precise limits on the stable region. The neutral curves are readily delineated, but the interpretation of their role as regards stability, is difficult. Numerical studies indicate stability within the neutral curves, and limit cycle behaviour outside. The Hopf theory regarding the existence of periodic solutions (or limit cycles) depends on a knowledge of the neutral curves for its application to this problem. Such analytic approaches are nonconstructive in that they only prove existence (and sometimes stability) of the limit cycles, but provide few accurate estimates of the period or amplitude.

For $f=g$, the numerical calculations of the limit cycles show some very fascinating properties of these stable solutions. At $\lambda= \pm i d_{0}$, the real part of the first eigenvalue passes from negative to positive, and the solution bifurcates. At the higher neutral roots further eigenvalues pass into the right-hand side of the complex eigenplane, and, for $\theta$ large enough, the limit cycle forms a loop; actually intersecting and crossing over itself. This behaviour corresponds to a splitting of the major peak of the curve $\eta=\eta(\tau)$. This behaviour is relevant to population dynamics for frequently the population density curves show some splitting of the peaks, see, for example, Pielou [20].

A more general differential-difference equation to model a single species can be formulated as

$$
\dot{\eta}(\tau)=-(1+\eta(\tau)) \sum_{i=1}^{n} f_{i} \eta\left(\tau-\theta_{i}\right), \quad 1=\theta_{1}<\theta_{2}<\cdots<\theta_{n},
$$

with $f_{t}, \theta_{1}$ constant. This model has $n$ different time lags, where the smallest lag has been normalised to 1 . Such an equation can be regarded as a more accurate approximation to a self-regulatory process in the population model (see Section 1). Qualitatively (5.1) has the same general properties as outlined earlier for the two time lag models (1.3). In fact the results (a) $-(\mathrm{g})$ in Theorem 1 can be carried across for (5.1), with $\theta$ replaced by $\theta_{n} ; f+g$ replaced by $\sum_{t=1}^{n} f_{i}$; and the conditions $f>0, g>0$ replaced by $f_{t}>0$ for all $i=1, \ldots, n$. Note that from Theorem 1(f), the upper bound on $\eta$ depends on the largest time lag; and from Theorem 2(g), the condition for the zeros to be unbounded depends on the smallest time lag.

The eigenvalue equation associated with (5.1) is

$$
\lambda=-\sum_{i=1}^{n} f_{i} e^{-\lambda \theta_{i}}
$$


For purely real roots, $\lambda=r$, and $f_{1} \neq 0$, this can be written as

$$
-r / f_{1}=e^{-r}+\sum_{i=1}^{n}\left(f_{i} / f_{1}\right) e^{-\lambda \theta_{1}}=H_{1}\left(r, f_{i}, \theta_{l}\right),
$$

where $H_{1}$ is a generalisation of the $H$ function defined in (2.3). Thus if $f_{t}<0$ for all $i=1, \ldots, n$, then (5.3) admits a real positive root, and so (5.1) is unstable to small perturbations. This result is independent of the time lags, and can also be obtained by Liapunov function techniques, see Bailey and Williams [1], see also Silkowski [21]. If $f_{i}>0$, for all $i$, then $H_{1} \geqslant 0$ for all $r$ and $\lim _{r \rightarrow-\infty} H_{1}\left(r, f_{t}, \theta_{l}\right)=$ $+\infty$, where the $\theta_{n}$ term dominates. Thus there is a critical surface, depending on $\theta_{n}$, on which there is a double real negative root. Equation (5.3) can also be written as

$$
-r=\sum_{i=1}^{n} f_{i} e^{-r \theta_{i}}=F\left(r, f_{i}, \theta_{i}\right)
$$

where $F\left(0, f_{i}, \theta_{t}\right)^{\cdot}=\sum_{t=1}^{n} f_{i}, \lim _{r \rightarrow \infty} F\left(r, f_{t}, \theta_{l}\right)=0$. So, considering (5.4) as the intersection of the straightline $-r$ and the continuous function, $F$, we find a purely real positive root if the value of $F\left(0, f_{l}, \theta_{l}\right)$ is negative. Thus if $\sum_{i=1}^{n} f_{t}<0$, then (5.3) admits a real positive root, and so (5.1) is unstable to small perturbations; this result is stronger than the one given above by consideration of $H_{1}$.

Purely imaginary roots of (5.2) in the case $f_{i}=f, \theta_{t}=i$, for all $i=1, \ldots, n$, can be calculated exactly, and show that the period of neutral oscillations, $2(n+1)$, is determined by the largest time lag; see also Kaplan and Yorke [11].

Complex solutions of (5.2) occur as conjugate pairs, with unstable eigenvalues having both real and (positive) imaginary parts bounded above by $\sum_{t=1}^{n}\left|f_{t}\right|$ (see Section 4). The imaginary part of (5.2) yields a generalisation of the rhombus condition namely if $\sum_{i=1}^{n}\left|f_{\imath}\right| \theta_{\imath}<1$ then there are no complex eigenvalues with positive real part.

Some of the results in Theorem 1 also carry over when a term without lag, $-f_{0} \eta(\tau)$, is included in the summation of (5.1). The search for purely real eigenvalues in this model leads to the equation

$$
-r-f_{0}=F\left(r, f_{i}, \theta_{\imath}\right),
$$

where $F$ is defined as in (5.4). Thus if $\sum_{t=1}^{n} f_{t}<0$, then there is a purely real eigenvalue, and the model is unstable to small perturbations. This result is conjectured in the paper by Bailey and Williams [1], but their Liapunov functions prove it only for $n=1$. They also show that if $f_{0}>0$ and $\sum_{i=1}^{n}\left|f_{i}\right|<f_{0}$, then the solution is stable. This model with $n=1$ has been studied in the ecological literature, Maynard Smith [15], and is discussed in Braddock and van den Driessche [2]. Gallucci (1980, personal communication) has indicated that this model $n=2$ is applicable in fisheries management, with reproduction and food regeneration lags. 


\section{Appendix}

A. Proof of Theorem 1. The proofs of parts (a), (c), (d) and (e) of Theorem 1 follows the methods outlined by Wright and the details are not repeated here. In considering (b), assume that $\eta(\tau) \rightarrow L$ which is a constant, as $\tau \rightarrow \infty$, then $\dot{\eta}(\tau) \rightarrow 0$ as $\tau \rightarrow \infty$ and $-L(f+g)(1+L)=0$, from (1.3). Thus 0 and -1 are the only possible finite limits as $\tau \rightarrow \infty$, provided that $f+g \neq 0$. If $f+g=0$, the limit is not determined by this method; in fact, for constant initial data $\eta(\tau)=$ $\eta(0),-\theta \leqslant \tau \leqslant 0$, the solution is $\eta(\tau)=\eta(0)$ for all $\tau$. This discussion illustrates the singular nature of the case considered by Cooke and Yorke [4].

In proving (f), observe that the zeros divide the $\tau$ axis into intervals on which $\eta(\tau)$ is either positive or negative. If, for example, $\eta(\tau)>0$ on such an interval, it attains its maximum at $\tau=\tau^{*}$, and $\dot{\eta}\left(\tau^{*}\right)=0$. Then, by (1.3),

$$
f \eta\left(\tau^{*}-1\right)+g \eta\left(\tau^{*}-\theta\right)=0,
$$

since $\eta(\tau) \neq-1$. If $f>0$ and $g>0$, then $\eta(\tau)$ changes sign for some $\tau_{1}$ such that

$$
\tau^{*}-\theta<\tau_{1}<\tau^{*}-1 \text {. }
$$

Dividing (1.3) by $1+\eta(\tau)$, and integrating from $\tau_{1}$ to $\tau^{*}$, we have

$$
\begin{aligned}
\ln \left(1+\eta\left(\tau^{*}\right)\right) & =-\int_{\tau_{1}-1}^{\tau^{*}-1} f \eta(s) d s-\int_{\tau_{1}-\theta}^{\tau^{*}-\theta} g \eta(s) d s \\
& <\theta(f+g),
\end{aligned}
$$

since $\eta(\tau)>-1$, and $1<\tau^{*}-\tau_{1}<\theta$. Hence

$$
\eta\left(\tau^{*}\right)<e^{\theta(f+g)}-1
$$

and this result holds for all maxima, so that for $\tau \geqslant \tau_{0}$ where $\tau_{0}$ is the smallest value of $\tau$ such that $\eta(\tau)=0$, then $\eta(\tau)$ is bounded below and above as stated in (f).

To prove (g), take $\eta(0)>-1, f>0, g>0$, and $f+g>1$, and assume that the zeros of $\eta(\tau)$ are bounded. Then, by (c) and (e), there exists $\tau_{2}$ such that $(f+g)(1+\eta(\tau))>1$, and $\eta(\tau)$ is of constant sign whenever $\tau>\tau_{2}$. Suppose the sign of $\eta$ is positive; then, for $\tau>\tau_{2}+0$, equation (1.3) gives

$$
\dot{\eta}(\tau)<-\frac{1}{(f+g)}(f \eta(\tau-1)+g \eta(\tau-\theta))<0 .
$$

So, by integrating from $\tau_{2}+2 \theta$ to $\tau_{2}+2 \theta+1$,

$$
\begin{aligned}
\eta\left(\tau_{2}+2 \theta+1\right)- & \eta\left(\tau_{2}+2 \theta\right)<-\frac{1}{(f+g)} \int_{\tau_{2}+2 \theta-1}^{\tau_{2}+2 \theta} f \eta(s) d s+\int_{\tau_{2}+\theta}^{\tau_{2}+\theta+1} g \eta(s) d s \\
& <-\frac{f}{f+g} \eta\left(\tau_{2}+2 \theta\right)-\frac{g}{f+g} \eta\left(\tau_{2}+\theta+1\right)<-\eta\left(\tau_{2}+2 \theta\right),
\end{aligned}
$$


which implies that $\eta\left(\tau_{2}+2 \theta+1\right)<0$, and gives a contradiction. A similar contradiction is reached if $\eta$ is negative. The zeros of $\eta$ are, therefore, unbounded, so $(\mathrm{g})$ is true.

In considering (h), we wish to determine if $\eta(\tau) \rightarrow 0$ as $\tau \rightarrow \infty$, and to determine the range of values of the parameters $f<0, g<0$ and $\theta$ for which this result holds. If the zeros of $\eta(\tau)$ are bounded, the result is obvious since $\eta(\tau) \rightarrow 0$ monotonically for sufficiently large $\tau$ (see Wright [25]).

Assume that the zeros are unbounded and let

$$
u=\lim _{\tau \rightarrow \infty} \eta(\tau), \quad v=-\lim _{\tau \rightarrow \infty} \eta(\tau) .
$$

Let $\varepsilon>0$ be any positive number such that

$$
-v-\varepsilon<\eta(\tau)<u+\varepsilon,
$$

for all $\tau$ sufficiently large. If $\tau^{*}$ is a maximum or minimum, then from (A.1),

$$
-\theta(f+g)(u+\varepsilon)<\ln \left(1+\eta\left(\tau^{*}\right)\right)<(v+\varepsilon) \theta(f+g),
$$

whence

$$
e^{-\alpha(u+\varepsilon)}-1<\eta\left(\tau^{*}\right)<e^{\alpha(v+\varepsilon)}-1,
$$

where $\alpha=\theta(f+g)$. With this definition of $\alpha,($ A.3) is identical with equation (3.3) of Wright [25]. The rest of Wright's work can be carried across and $\eta(\tau) \rightarrow 0$ as $\tau \rightarrow \infty$, for $0<\alpha \leqslant 1$. Thus the proof of Theorem 1 is complete.

B. Proof of Theorem 3. In part (a) of Theorem 3, the values of $f$ are restricted to the interval $\left(0, f_{c}(\theta)\right)$, and the bounds on $\beta$ imply that if $\alpha \leqslant 0$, then $0 \leqslant \beta \leqslant 2 f_{c}$ $=d_{c}(\theta) / \sin d_{c}(\theta)<\pi / 2$, since $d_{c}(\theta)<\pi / 2$. Thus all eigenvalues for which $\beta>2 f_{c}(\theta)$ have a negative real part; those for which $\alpha \geqslant 0$ must have $0 \leqslant \beta \leqslant$ $d_{c}(\theta) / \sin d_{c}(\theta)$. In this range, $\cos \beta>0$ so that we can consider $(2.10)$ in the form $-\alpha /(f \cos \beta)=H(\alpha, \gamma, \theta)$ (compare the discussion of the purely real roots). Now, for $\alpha \geqslant 0$ and $\gamma \geqslant-1, H(\alpha, \gamma, \theta) \geqslant 0$ for all $\theta$. If $\gamma<-1$, then $H(\alpha, \gamma, \theta) \leqslant 0$ for $0 \leqslant \alpha \leqslant \alpha_{0}$ where $\alpha_{0}=\ln (-\gamma) /(\theta-1)$, and $H(\alpha, \gamma, \theta) \geqslant 0$ for $\alpha \geqslant \alpha_{0}$. For $\gamma \geqslant-1$, the straight line $-\alpha /(f \cos \beta)$, intersects $H(\alpha, \gamma, \theta)$ at points for which $\alpha<0$; so the eigenvalues have negative real parts. The condition $\gamma \geqslant-1$ gives $\cos \beta \theta+\cos \beta \geqslant 0$, and equality occurs when $\beta \in\left\{d_{n}(\theta), d^{m}(\theta) ; m, n \in J\right\}$. Obviously $\gamma \geqslant-1$ for $0 \leqslant \beta \leqslant d_{c}(\theta)$, and we need to consider the sign changes of $\gamma+1$ for $d_{c}(\theta) \leqslant \beta \leqslant d_{c}(\theta) / \sin d_{c}(\theta)$. Note that this phase of the proof does not depend on whether $f_{<} f_{c}$, so that wherever $\cos \beta>0, \gamma \geqslant-1$, all the eigenvalues have negative real parts.

On those intervals of the $\beta$ axis $\left(d_{c}(\theta) \leqslant \beta \leqslant d_{c}(\theta) / \sin d_{c}(\theta)\right)$ for which $\gamma<-1$, equation (2.10) permits a root $\alpha \geqslant 0$. We now show that equation (2.11) cannot have a root with $\alpha \geqslant 0$, for $\gamma<-1$, and that the condition $0<f<f_{c}$ is 
necessary for the proof. To do this, we turn briefly to the positive roots $d_{n}(\theta)$ and $d^{m}(\theta)$ defined in Section 2, and note that $d_{n}(\theta)-d_{n-1}(\theta)=2 \pi /(\theta+1), d^{m}(\theta)$ $-d^{m-1}(\theta)=2 \pi /(\theta+1)$, so that the difference in length of these intervals is $4 \pi /(\theta-1)(\theta+1)$. Now the first $p$ roots of each class will alternate (or interleave) provided that $p 4 \pi /(\theta+1)(\theta-1) \leqslant 2 \pi /(\theta+1)$, where $2 \pi /(\theta+1)$ is the shorter interval between members of the same class. This requires that $p \leqslant$ $[(\theta-1) / 2]+1=p^{*}$, where $p$ is an integer. For $p \geqslant p^{*}, d_{p}(\theta) \geqslant \pi / 2$ and $d^{P}(\theta) \geqslant \pi / 2$. Hence for $d_{c} \leqslant \beta \leqslant d_{c} / \sin d_{c}$, the roots interleave, giving a finite set $S=\left\{\left(d_{n}(\theta), d^{n}(\theta)\right), n \in J\right\}$ of intervals on which $\gamma<-1$.

Now consider (2.11) in the form

$$
G(\alpha, \beta, \theta, f)=\beta-f\left(e^{-\alpha} \sin \beta+e^{-\alpha \theta} \sin \beta \theta\right) .
$$

Since $\sin \left(\theta d_{n}(\theta)\right)=\sin d_{n}(\theta)$, then for $\alpha \geqslant 0$,

$$
\begin{aligned}
G\left(\alpha, d_{n}, \theta, f\right) & =d_{n}-\left(e^{-\alpha}+e^{-\alpha \theta}\right) f \sin \left(d_{n}\right), \\
& \geqslant d_{n}\left(1-f / f_{n}\right) .
\end{aligned}
$$

Thus $G\left(\alpha, d_{n}, \theta, f\right)>0$ if $0<f<f_{c}(\theta)$, as $f_{c}(\theta)<f_{n}(\theta)$ for all $n$, and equality occurs only when $f=f_{c}$ and $n=0$; i.e. at the first neutral root. The condition $\gamma<-1$ implies that $-\cos \beta \theta>\cos \beta>0$ for $d_{c} \leqslant \beta \leqslant 2 f_{c}$, and

$$
\frac{\partial G}{\partial \beta}(\alpha, \beta, \theta, f) \geqslant 1-f e^{-\alpha} \cos \beta \text {. }
$$

For $\alpha \geqslant 0$, and since $f<f_{c} \leqslant \pi / 4, \partial G / \partial \beta>0$ whenever $\gamma<-1$. Thus $G$ is a monotonic increasing function of $\beta$ on those intervals of the $\beta$ axis for which $\gamma<-1$, and hence $G>0$ on those intervals. Therefore, there can be no eigenvalues with $\alpha \geqslant 0$ for $\gamma<-1$ and so part (a) of the stability proof is complete.

In discussing the instability for $f \geqslant f_{c}(\theta)$, in (b), we consider where and how the above proof of (a) fails. The breakdown in the proof near $f_{c}$ comes from the sign of $G\left(\alpha, d_{n}, \theta, f\right)$ for $\alpha>0$ in (B.1). For $n \in J$ and $n \neq 0$, we have $f_{c}=f_{0}<f_{n}$. Let $f=f_{0}+\varepsilon$, where $\varepsilon \geqslant 0$ and small enough so that $f_{0}=f_{c} \leqslant f<f_{1}$. Then $G\left(0, d_{0}, \theta, f\right) \leqslant 0$ while $G\left(\alpha, d_{n}, \theta, f\right)>0$ for $\alpha \geqslant 0$ and $n \in J$ with $n \neq 0$. From (2.3) the eigenvalues $\lambda=\lambda(f, \theta)$ depend continuously on the parameters $f$ and $\theta$. At $\varepsilon=0, \lambda=\lambda\left(f_{0}, \theta\right)=i d_{0}$ is an eigenvalue. For $\varepsilon>0$ and small, continuity requires that the corresponding eigenvalue $\lambda=\lambda\left(f_{0}+\varepsilon, \theta\right)=\alpha_{1}+i \beta_{1}$ will be near $\lambda\left(f_{0}, \theta\right)$. The above discussion on the behaviour of $(2.10)$ and (2.11) indicates that there is such an eigenvalue with $\alpha_{1}>0$ and $\beta_{1}>d_{0}$. This completes the proof of (b) and indicates that the nonlinear equation (3.1) is unstable for $f>f_{c}(\theta)$, and that there is a neutral root of the linear equation (3.2) for $f=f_{c}(\theta)$.

The above argument can be extended to consider the behaviour of certain of the eigenvalues as the value of the parameter passes through the higher neutral values $f_{1}, f_{2}, \ldots$, in turn. At $f=f_{n}(\theta)$, we know that there is a pure imaginary 
eigenvalue $\lambda\left(f_{n}, \theta\right)=i d_{n}$. As the sign of $f-f_{n}$ goes from negative to positive, the sign of $G\left(0, d_{n}, \theta, f\right)$ reverses from positive to negative and there is an eigenvalue $\lambda(f, \theta)=\alpha_{n}+i \beta_{n}$ such that $\alpha_{n}>0$ and $\beta_{n}>d_{n}$. Thus another conjugate pair of eigenvalues pass through the imaginary axis and into the right-hand half plane as the value of the parameter $f$ takes values in the set $\left\{f_{n}\right\}$.

C. Properties of the real roots for general $f$ and $g$. The roots of (4.1) are obtained from the interactions of a straight line $r / f$, and the function $H(r, \gamma, \theta)$. The parameter $\theta$ plays little part in the following discussion. However, the structure of the discussion pivots on the values taken by $\gamma$ and $f$, so that the details are given in 4 parts.

C.1. $\gamma \geqslant 0$. For this range of $\gamma, H(r, \gamma, \theta) \geqslant 0$, and is monotonically decreasing for all values of $r$. The behaviour of $H(r, \gamma, \theta)$ is quantitatively the same as that of $H(r, \gamma=1, \theta)$, see Figure 1. Arguing as in Section 2, we thus have the following results for (4.1).

(a) If $f<0$, then $g \geqslant 0$, the gradient of the straight line is positive and there is a single real positive root $r_{+}$such that $r_{+} \rightarrow+\infty$ as $f \rightarrow+\infty$. Equation (1.3) is unstable to small oscillations.

(b) If $f>0$, then $g \leqslant 0$, and there can be one, two, or no negative roots according as to whether the straight line $-r / f$ is tangent to $H$, cuts $H$, or does not intersect it. The conclusions given in Theorem 2(c) again apply with the critical root $r=r_{K}$ and corresponding $f=f_{K}$ determined through the tangency equations

$$
\left.\frac{d H}{d r}(r, \gamma, \theta)\right|_{r_{K}}=\frac{H\left(r_{K}, \gamma, \theta\right)}{r_{K}}=-\frac{1}{f_{K}} .
$$

Note that when $g=0, f_{K}=e^{-1}$, the single time lag results.

C.2. $-1<\gamma<0$. In this second case, and in fact for all $\gamma<0$, $\lim _{r \rightarrow \infty} H(r, \gamma, \theta)=-\infty$. Further, $H(0, \gamma, \theta)=1+\gamma>0$ for $\gamma>-1$, and $H(r, \gamma, \theta) \geqslant 0$ for $r \geqslant 0$. The function $H(r, \gamma, \theta)$ has its zero at $r=r_{z}=$ $(\theta-1)^{-1} \ln (-\gamma)$, so that $r_{z}<0$ for $-1<\gamma<0$. The function $H$ attains its maximum at $r=r_{\max }=(\theta-1)^{-1} \ln (-\theta \gamma)$ so that $r_{\max } \leqq 0$ according to $\gamma \theta \leqq-1$. The point of inflection of $H$ is at $r=r_{\text {inf }}=(\theta-1)^{-1} \ln \left(-\theta^{2} \gamma\right)$, and $r_{\text {inf }} \leqq 0$ according to $\theta^{2} \gamma \lesseqgtr-1$. Figure 9 indicates the behaviour of $H(r, \gamma, \theta)$ for $\theta=2$ and various representative values of $\gamma$, particularly $\gamma=-1 / 2$, for which $r_{z}=-\ln 2$, $r_{\max }=0$, and $r_{\text {inf }}=\ln 2$. Considering the intersection of $H(r, \gamma, \theta)$ the straight line $-r / f$, gives the following results:

(a) If $f>0$, then $g<0$, and there is a single real negative root $r_{\text {- }}$ such that $r_{z}<r_{-}<0$. Further, $r_{-} \rightarrow r_{z}$ as $f \rightarrow+\infty$, and $r_{-} \rightarrow 0^{-}$as $f \rightarrow 0$. 
(b) If $f<0$, then $g>0$, and there are two roots $r_{1}>0$ and $r_{2}<r_{z}<0$. Here, $r_{1} \rightarrow \infty$ and $r_{2} \rightarrow r_{z}$ as $f \rightarrow-\infty$; and $r_{1} \rightarrow 0$, and $r_{2} \rightarrow-\infty$ as $f \rightarrow 0^{-}$. Thus there is always a positive real root for (4.1), and equation (1.3) is unstable to small perturbations.

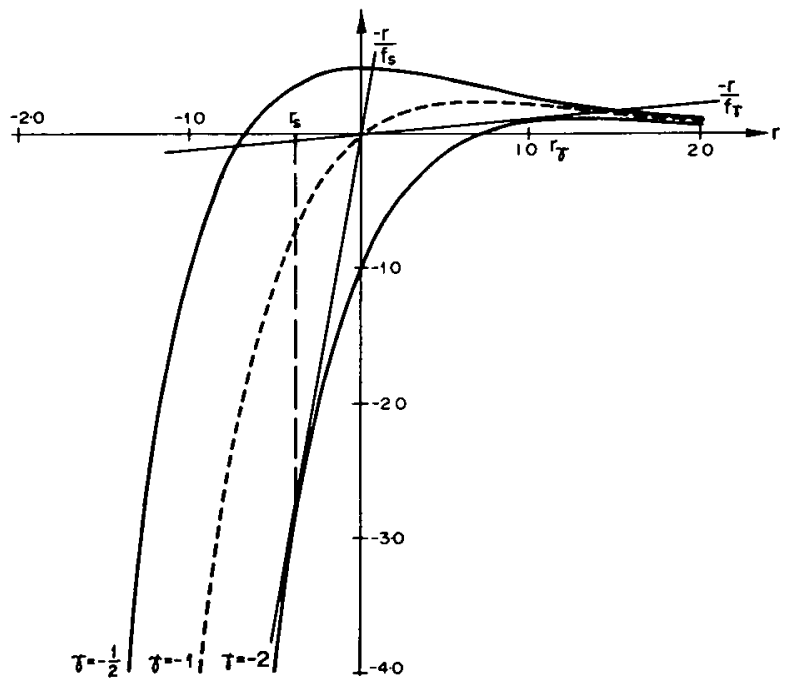

Figure 9. Graphs of $H(r, \gamma ; 2)$ for $\gamma=1 / 2,-1,-2$; together with the tangent lines given the roots $r_{S}, r_{T}$.

C.3. $\gamma=-1$. The case introduces certain complications since $r_{z}=0$, and $r=0$ is always a root of (4.1), although other roots can occur (see Figure 9). Further, $d H(0,-1, \theta) / d r=\theta-1$, and we have the following results for the intersections of $-r / f$ and $H(r,-1, \theta)$ :

(a) If $f>0$, there are no other roots.

(b) If $f<0$, then there is the possibility of either an additional positive or negative root.

(i) If $f=-(\theta-1)^{-1}$, then $-r / f$ is tangent to $H(r,-1, \theta)$ at $r=0$, and it is readily shown that this is the only root.

(ii) If $-(\theta-1)^{-1}<f<0$, then there is a single root $r_{-}<0$ such that $r_{-} \rightarrow 0$ as $f \rightarrow-(\theta-1)^{-1}$ from above, and $r_{-} \rightarrow-\infty$ as $f \rightarrow 0^{-}$.

(iii) If $f<-(\theta-1)^{-1}$, then there is a single positive root $r_{+}$such that $r_{+} \rightarrow+\infty$, as $f \rightarrow-\infty$, and $r_{+} \rightarrow 0^{+}$as $f \rightarrow 0^{-}$. The existence of this root implies that (1.3) is unstable to small perturbations.

The above results are for the line $f+g=0$, and they are identical with those obtained by Cooke and Yorke [4]. 
C.4. $\gamma<-1$. In this final case $H(0, \gamma, \theta)<0$, and in fact, the zero crossing $r_{z}>0$. The qualitative behaviour of $H(r, \gamma, \theta)$ is shown in Figure 9 , for $\gamma=-2$, $\theta=2$; all $\theta>1$ and $\gamma<-1$ lead to a similar diagram. On considering the intersections of $-r / f$ and $H(r, \gamma, \theta)$, we then have the following results:

(a) If $f>0$, then there is a single positive root $r_{+}$such that $0<r_{+}<r_{z}$, and (1.3) is unstable to small oscillations.

(b) If $f<0$, there can be none, one or two positive or negative roots, denending on the magnitude of $f$. There is a critical value $r=r_{s}<0$, and a corresponding $f=f_{S}$, at which the straight line $-r / f_{S}$ is tangent to $H(r, \gamma, \theta)$ at $r=r_{S}$; the critical values $r_{s}$ and $f_{s}$ being determined by the tangency conditions (C.1). This critical case is obtained for $|f|$ small, but for $|f|$ large, there is the possibility of a positive critical point $r=r_{T}$ such that $r_{z}<r_{T}<r_{\max }$, and $f=f_{T}$. Then the line $-r / f$ is tangent to $H(r, \gamma, \theta)$ at $r=r_{T}$, and the critical values are determined from (C.1), the tangency condition. Since $H(r, \gamma, \theta)$ is strictly increasing for $-\infty<r<$ $r_{\max }$, the tangency condition implies that $f_{T}<f_{S}$.

C.5. Comments. The equation (C.1) is readily solved using Newton's method to obtain the critical roots, $r_{K}, r_{S}$ and $r_{T}$, and corresponding values $f_{K}, f_{S}$ and $f_{T}$, as functions of $\theta$ and $\gamma$. For each $f_{K}, f_{S}, f_{T}$, calculated for a given value of $\gamma$, we can define $g_{K}=\gamma f_{K}, g_{S}=\gamma f_{S}$, and $g_{T}=\gamma f_{T}$, and these curves are shown in Figure 8 for $\theta=2$. Observe that the curve $\left(f_{S}, g_{S}\right)$ originates from the point $(\theta-1)^{-1}(-1,1)$ and meets the $g$-axis at the point $\left(0, e^{-1} \theta^{-1}\right)$ passing smoothly across the axis and linking with the $\left(f_{K}, g_{K}\right)$ curve. The $\left(f_{K}, g_{K}\right)$ curve ends on the $f$-axis at the point $\left(e^{-1}, 0\right)$ as expected from Wright's results. The $\left(f_{T}, g_{T}\right)$ curve also starts from $(\theta-1)^{-1}(-1,1)$ but as $\gamma \rightarrow-\infty, f_{T} \rightarrow 0^{-}$and $g_{T} \rightarrow+\infty$.

\section{Acknowledgement}

The authors thank Hugh Barclay for discussions on biological aspects of this study. This work was supported in part by NRC grant A5233.

\section{References}

[1] H. R. Bailey and M. Z. Williams, "Some results on the differential-difference equation $x(t)=\sum_{t=0}^{N} A_{i} x\left(t-T_{t}\right)$ ", J. Math. Anal. Appl. 15 (1966), 569-587.

[2] R. D. Braddock and P. van den Driessche, "A population model with time delay", Math. Sci. 5 (1980), 55-65.

[3] J. R. Claeyssen, "Effect of delays on functional differential equations", J. Differential Equations 20 (1976), 404-440. 
[4] K. L. Cooke and J. A. Yorke, "Some equations modelling growth processes and gonorrhea epidemics", Math. Biosci. 16 (1973), 75-101.

[5] G. Dunkel, "Single species model for population growth depending on past history", in Seminar on Differential Equations and Dynamical Systems, Lecture Notes in Math. 60 (SpringerVerlag, New York, 1968).

[6] J. K. Hale, Theory of functional differential equations, Appl. Math. Sci. 3 (Springer-Verlag, New York, 1977).

[7] J. K. Hale, "Behaviour near constant solutions of functional differential equations", $J$. Differential Equations 15 (1974), 278-294.

[8] J. K. Hale, "Nonlinear oscillations in equations with delays", in Lectures in Appled Math. 17, (Amer. Math. Soc. 1979).

[9] G. E. Hutchinson, "Circular causal systems in ecology", Ann. N.Y. Acad. Sci. 50 (1948), $221-246$.

[10] G. S. Jones, "On the nonlinear differential difference equations $f^{\prime}(x)=-\alpha f(x-1)(1+f(x))$ ", J. Math. Anal. Appl. 4 (1962), 440-469.

[11] J. L. Kaplan and J. A. Yorke, "Ordinary differential equations which yield periodic solutions of differential delay equations", J. Math. Anal. Appl. 48 (1974), 317-324.

[12] J. L. Kaplan and J. A. Yorke, "On the stability of a periodic solution of a differential delay equation", SIAM J. Math. Anal. 6 (1975), 268-282.

[13] R. L. Kitching, "Time, resources and population dynamics in insects", Austral. J. Ecol. 2 (1977), 31-42.

[14] R. M. May, Stability and complextty in model ecosystems (Princeton Univ. Press, Princeton, 1973).

[15] J. Maynard Smith, Models in Ecology (C.U.P., Cambridge, 1974).

[16] R. D. Nussbaum, "Periodic solutions of some nonlinear autonomous functional differential equations", Ann. Mat. Pura Appl. 101 (1974), pp. 263-306.

[17] R. D. Nussbaum, "Differential delay equations with two time lags", Mem. Amer. Math. Soc. No. 205, Vol. 16(1) (1975), 1-62.

[18] R. D. Nussbaum. "Periodic solutions of non-linear autonomous functional differential equations", Lecture Notes in Math. 730 (Springer-Verlag, New York, 1979), 283-325.

[19] E. C. Pielou, An introduction to mathematical ecology (Wiley, New York, 1969).

[20] E. C. Pielou, Population and community ecology (Gordon and Breach, New York, 1974).

[21] R. Silkowski, "A star-shaped condition for stability of linear retarded functional differential equations", Proc. Roy. Soc. Edınburgh Sect. A 83 (1979), 189- 198.

[22] H. Stech, "The effect of time lags on the stability of the equilibrium state of a population growth equation", J. Math. Biol. 5 (1978), 115-130.

[23] H. Stech, "The Hopf bifurcation: a stability result and application", J. Math. Anal. Appl. 71 (1979), 525-546.

[24] $\mathrm{H}-\mathrm{O}$. Walther, "Existence of a non-constant periodic solution of a non-linear autonomous functional differential equation representing the growth of a single species population", $J$. Math. Biol. 1 (1975), 227-240.

[25] E. M. Wright, "A non-linear difference-differential equation”, J. Reine Angew. Math. 194 (1955), 66-87. 